\title{
Interactive Analysis of Event Data Using Space-Time Cube
}

\author{
Peter Gatalsky, Natalia Andrienko, and Gennady Andrienko \\ Fraunhofer Institute for Autonomous Intelligent Systems \\ Schloss Birlinghoven, D-53754 Sankt Augustin, Germany \\ http://www.ais.fraunhofer.de/ gatalsky \\ \{gatalsky@ais.fraunhofer.de,gennady.andrienko@ais.fraunhofer.de\}
}

\begin{abstract}
In exploratory data analysis, the choice of tools depends on the data to be analyzed and the analysis tasks, i.e. the questions to be answered. The same applies to design of new analysis tools. In this paper, we consider a particular type of data: data that describe transient events having spatial and temporal references, such as earthquakes, traffic incidents, or observations of rare plants or animals. We focus on the task of detecting spatio-temporal patterns in event occurrences. We demonstrate the insufficiency of the existing techniques and approaches to event exploration and substantiate the need in a new exploratory tool. The technique of spacetime cube, which has been earlier proposed for the visualization of movement in geographical space, possesses the required properties. However, it must be implemented so as to allow particular interactive manipulations: changing the viewing perspective, temporal focusing, and dynamic linking with a map display through simultaneous highlighting of corresponding symbols. We describe our implementation of the space-time cube technique and demonstrate by an example how it can be used for detecting spatio-temporal clusters of events.
\end{abstract}

Keywords: space-time-cube, spatio-temporal data, exploratory data analysis, data visualization

\section{Introduction}

This paper deals with some issues of exploratory analysis of spatio-temporal data, that is, data having both spatial and temporal references. In particular, we focus on the analysis of events - discrete spatial objects having relatively short life duration. Examples of events are earthquakes, traffic incidents, occurrences of diseases, or observations of rare animals. An important question arising in analysis of event data is how the events are distributed in space and time. A proper data representation is needed for answering this question.

In early 70s Hägerstrand [1] developed a graphic view on time as an additional spatial dimension. He suggested a three-dimensional diagram, the so-called space-time cube, to show life histories of people and how people interact in space and time. The base of the cube represents the two-dimensional geographical space while the cube's height represents the temporal dimension. At the time when the concept was introduced, the options for creating such graphics were limited to manual methods. This was an obvious limitation of the approach, since creation of each new diagram was a laborious drawing exercise. Today, when modern computer technologies provide much better opportunities for data visualization, the interest of researchers to Hägerstrand's time-geography has revived [2]. Prominent application examples have been made in ITC, Enschede, the Netherlands [3]. Thus, Figure 1 shows the representation of the Napoleon's Russian campaign in 1812. Summarizing all experiments carried out with space-time cube, a project of dynamic visualization environment has been proposed [4].

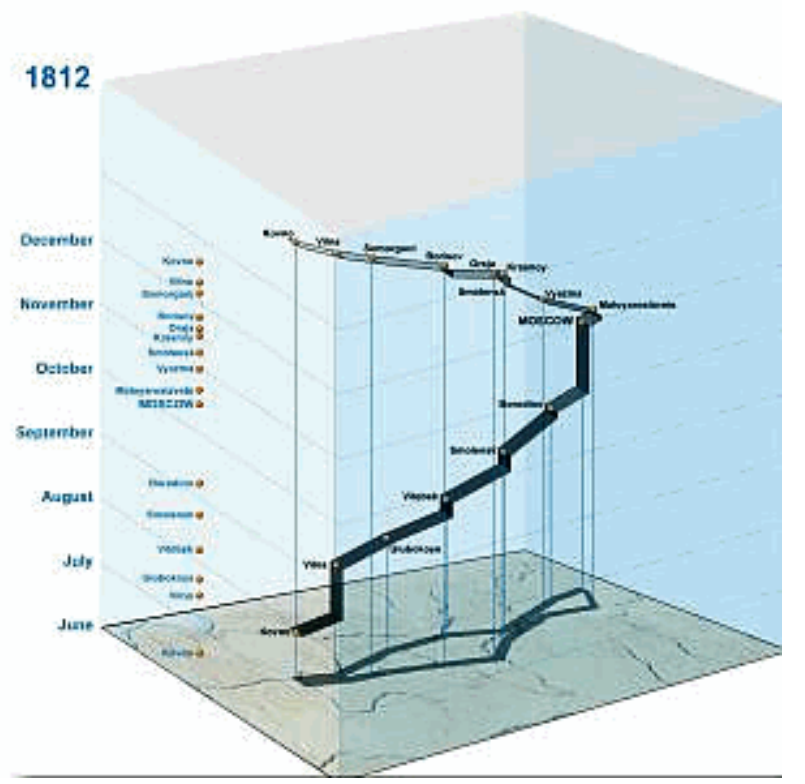

Figure 1. Representation of object movement in a "space-time cube". (Source:[4])

Both Hägerstrand and the research team from ITC applied space-time cube to data concerning movement of objects, i.e. changes of spatial locations. In this paper, we 
propose an application of Hägerstrand's concept of cube to a different data type, specifically, data about events.

The remainder of the paper is built as follows. In the second section, we introduce our task-analytic approach to the design of tools for visual exploratory analysis of spatio-temporal data. We demonstrate that current approaches to event representation are insufficient and there is a need in a new instrument that could properly support the exploration of spatio-temporal event patterns. This motivated our attempts to apply the idea of spacetime cube to event data. The third section describes our implementation of an interactive tool for data analysis involving data representation in a space-time cube. In the fourth section, we consider an example of application of the tool to the search of spatio-temporal clusters of earthquake occurrences. Finally, we draw some conclusions and indicate possible directions for future work.

\section{Task-analytical approach to data visualisation}

Effectiveness of data analysis strongly depends on the selection of proper tools. The right choice, in turn, depends on the characteristics of the data and on the analysis tasks that need to be fulfilled, or, in other words, the questions to be answered. The same applies to the design of new exploratory tools: data and task characteristics define the space of design alternatives and the directions for search.

In [5], we suggested an operational typology of spatio-temporal data and tasks that can potentially arise in exploratory analysis of such data. On the basis of this typology, we cataloged the existing tools and techniques for visualization and exploration of spatio-temporal data, i.e. we categorized the tools according to the types of data and tasks they are suitable for. Here, we give a brief account of the typology. This will help us to define the typical tasks arising in analysis of event data, substantiate the need for a tool like space-time cube, and compare the tool with other tools and techniques suggested for event analysis.

\subsection{Data and tasks}

According to Peuquet [6], spatio-temporal data involve three major components: space (where), time (when), and objects (what). Each component consists of specific elements that may have some properties (attributes) and be linked by various relationships. A slightly different division can be found in cartographic literature, where spatial, temporal, and thematic aspects of geographical phenomena are distinguished [7]. The term "thematic" covers non-spatial and non-temporal properties of geographical objects or phenomena.
In choosing methods for analysis of spatio-temporal data, their spatial, temporal, and thematic characteristics play an essential role. Let us briefly enumerate the most important distinctions. With respect to the spatial properties, objects or phenomena are often classified into points, lines, areas, and volumes [8]. Another typology of spatial phenomena is based on two orthogonal dimensions: spatial continuity and spatial (in) dependence [7][8]. According to the first dimension, phenomena are characterised as discrete or continuous. Discrete phenomena occur at isolated locations while continuous phenomena occur everywhere. According to the second dimension, phenomena may be classified as smooth (adjacent locations are dependent) or abrupt (adjacent locations are independent). Smooth phenomena change in a gradual fashion while abrupt phenomena change suddenly. Events can be characterized as discrete and abrupt phenomena. They are most often considered as points in space.

Temporal characteristics are related to the existence of objects/phenomena at different time moments and variation of their spatial and thematic properties over time. Geographic phenomena may undergo different types of changes [9]: existential changes (appearing and disappearing), changes of spatial properties (location, shape, size, orientation, altitude, height, gradient, and volume), and changes of thematic properties, which include qualitative changes and changes of ordinal or numeric characteristics (increase and decrease). Sometimes only one type of changes takes place (or is of interest for an analyst), but in many cases one needs to consider two or three types simultaneously. In event data, existential characteristics are of primary interest, i.e. when the events appear.

Attributes, i.e. thematic components of spatially referenced data, are typically distinguished according to levels of measurement introduced by Stevens [10]: nominal, ordinal, interval, and ratio. Often the categories "interval" and "ratio" are united into a single category "quantitative", or "numeric" [11][7]. Events may have various properties expressed by different types of attributes. For example, such events as observations of rare insects may be described through the following attributes: the species of the observed insect (a nominal attribute); the stage of development: egg, larva, pupa, or adult (an ordinal attribute); the number of individuals encountered (a numeric attribute).

Like the data characteristics, the tasks (questions) arising in analysis of spatio-temporal data have also much to do with the three major components of these data, i.e. space, time, and objects with their thematic properties. According to Peuquet, there are three possible types of questions concerning spatio-temporal data ([6], p.448): 
- when + where $\rightarrow$ what: Describe the objects or set of objects that are present at a given location or set of locations at a given time or set of times.

- when + what $\rightarrow$ where: Describe the location or set of locations occupied by a given object or set of objects at a given time or set of times.

- where + what $\rightarrow$ when: Describe the times or set of times that a given object or set of objects occupied a given location or set of locations.

This classification corresponds to the notion of question types introduced by Jacques Bertin: "There are as many types of questions as components in the information" ([11], p.10). Another division of questions proposed by Bertin is according to levels of reading: elementary, intermediate, and overall. Elementary questions refer to individual elements of data (e.g. individual places, time moments, and objects) while questions of the intermediate and overall levels address more general characteristics of a phenomenon, e.g. how it is distributed in space, how it behaves in time, or how characteristics are distributed over a set of objects.

Koussoulakou and Kraak [12] demonstrate that, in the case of spatio-temporal data, the distinction according to the reading levels can be independently applied to the spatial and to the temporal dimensions of the data. For example, the question "What is the trend of changing values at location l?" belongs to the elementary level in relation to the spatial component and to the overall level with respect to the temporal component. An analogous observation can also be made for the object dimension.

Hence, each of the Peuquet's general question schemes of the form $\mathrm{A}+\mathrm{B} \rightarrow \mathrm{X}$ (where $\mathrm{A}$ and $\mathrm{B}$ denote known, or given, data components and $\mathrm{X}$ stands for unknown information) can be further subdivided according to the level on which the known information is specified: elementary $\mathrm{A}$ and $\mathrm{B}$, elementary $\mathrm{A}$ and overall/intermediate $\mathrm{B}$, overall/intermediate $\mathrm{A}$ and elementary $\mathrm{B}$, and overall/intermediate $\mathrm{A}$ and $\mathrm{B}^{1}$.

The focus of this paper is analysis of event data on the overall level with respect to both space and time. The corresponding question can be formulated as "What is the pattern of spatial and temporal distribution of the events and their thematic properties?" This is a specialization of the when + where $\rightarrow$ what question formula, in which when (time) and where (space) are considered on the overall level. Let us look which data visualization techniques are suitable for event data and to what extent they can help in answering this type of question.

\footnotetext{
1 In our opinion, there is no principal difference between the intermediate and overall levels, as defined by Bertin. Both levels involve consideration of sets rather than individual elements. The difference is whether the whole set or its subsets are considered.
}

\subsection{Techniques for event exploration}

Like any spatial objects, events can be represented on a map. Maps are indispensable in investigation of spatial patterns of event occurrence. For a static map, there are two possibilities: either all events are shown irrespective of the time of their occurrence or only events that took place at a selected moment or during a selected interval. In the first variant, the information about the time of event occurrences can be shown on the map using appropriate cartographic representation methods. Thus, in the system SpaTemp [13], the time of appearing of an event or the period of its existence can be indicated by a label placed on a map according to the location of the event. The "ages" of events may be represented by variation of colors. However, labels are suitable only for elementary tasks since they do not foster an integral view of the spatio-temporal distribution of the events. Color variation may be helpful when the events are not too numerous and there is no occlusion of the symbols that represent the events on the map. Under these conditions, concentrations of symbols similar in their colors correspond to spatio-temporal event clusters, which deserve particular attention as signs of possible event interactions. However, clusters can only be detected if events never re-occurred around the same place after a significant lapse of time, otherwise, even if there is no symbol occlusion, the mix of differently colored symbols obstructs seeing clusters. Besides, representing temporal information by symbol colors reduces the opportunities for displaying thematic characteristics of events.

The second approach, when only a portion of data corresponding to a selected time moment or interval is represented on a map, can be used only in combination with other techniques that compensate the information loss. One of them is map iteration, i.e. juxtaposition of several maps where each map shows events pertinent to a different time moment or interval. Obviously, the number of perceptible images that can be simultaneously shown on a computer screen is limited, and, hence, long time periods have to be investigated at a rather coarse temporal resolution. As a result, spatially close event symbols on a single map may not necessarily signify temporal closeness. On the other hand, temporally close events can be occasionally split between two maps.

Another possibility is to combine the display of event subsets with querying, i.e. interactive tools for subset selection. In particular, the user should be able to choose various time moments and intervals, and the map must be automatically updated to represent the corresponding events. We would like to mention some interesting implementations of controls for choosing times that combine the linear and cyclical views of time $[14][15][16][17]$.

A specific form of temporal filtering of event data occurs in the course of map animation. At each display 
moment, the map shows events that took place at some real time moment or during some interval. This moment or interval is shifted forth or back in time, and the map is correspondingly updated.

Temporal filtering, either through querying or through animation, is very helpful when events are numerous. However, the user sees only a portion of data (a time slice) at each moment. In order to get an overall picture of spatio-temporal distribution, the user has to memorize many slices and mentally integrate them. Another difficulty is to select an appropriate temporal resolution. If the map shows only events that occurred at some moment or during a very short interval, the events may be too sparse for observing any clusters. If the interval is too long, "false" clusters may appear. Even if the interval is neither too short nor too long, there may be clusters stretching over several time slices. This causes difficulties in estimating their spatial and temporal extents and the number of events involved. Besides, while it is relatively easy to focus the attention on a single place where several events co-occur, it may be problematic to keep the eye on the whole territory.

Among the techniques suggested for dealing with a large number of events is data aggregation. The tool described in [15] supports various ways of data aggregation: spatial, temporal, and categorical (i.e. according to types of the events). The software displays summary characteristics of the aggregates, such as the total number of events or their average duration, and allows the user to "drill down" into each aggregate in order to see data about the individual events. The summary data about spatially aggregated events (e.g. traffic incidents aggregated by road fragments) are shown on an interactive map by symbols the size of which is proportional to the number of events. Two different types of temporal aggregation are supported: by days of week and by calendar dates. In the first case, aggregates unite all traffic incidents that occurred on the same day of the week irrespective of calendar dates. The aggregates are represented by bars on a bar chart with the height of a bar proportional to the number of the events. In the second case, events that occurred on the same date are grouped together and shown on a calendar display using square symbols with sizes proportional to the numbers of incidents. It may be noticed that spatial aggregation entails a loss of the temporal information, and vice versa. Hence, this technique is unsuitable for exploring spatio-temporal distribution, although it can provide valuable insights concerning the distribution of events in space or time taken separately.

There is a principal possibility to reduce information losses resulting from aggregation by applying temporal aggregation not to the whole territory but to its parts. Thus, in the case of traffic incidents, one could build a bar chart or line graph representing the variation of the number of event occurrences over time for each road fragment. This would allow the user to compare temporal patterns of event occurrences at different places. However, this only provides a sort of "space slices" analogous to time slices resulting from temporal filtering. From such space slices, one can gain knowledge concerning temporal grouping of events at different places but not concerning spatial grouping of events close in time.

\subsection{Need for the cube}

As we have demonstrated, none of the approaches suggested thus far for event visualization and analysis sufficiently supports the task of searching for spatiotemporal patterns. This task requires an overall view of both space and time, which is difficult to reconstruct from spatial or temporal slices. Hence, there is a need in a tool capable to represent simultaneously the whole space-time continuum and the positions of events in this continuum. The idea of space-time cube is an obvious response to this need.

In the examples cited in the introduction, space-time cube is used to represent data about spatial movements and spatial interaction, i.e. changes of spatial properties of persistent discrete objects. According to this technique, points in three-dimensional space, where the vertical dimension corresponds to time, represent the positions of an object at different time moments. Lines connect the points corresponding to consecutive moments. Besides trajectories of object movement, a cube display allows the user to explore the speed of movement. In this representation, gently sloping path segments indicate fast movement, i.e. long distance in space traveled in short time, while steep segments correspond to slow motion. Vertical lines occur when an object stays for some time period in the same place. Hence, the cube exposes certain patterns that provide valuable information about characteristics of object movement. One may expect that representation of events in a cube will also foster detection of interesting patterns. However, events are a different type of phenomenon as compared to object movement, and the types of patterns to look for are also different.

\section{Interactive space-time cube display in CommonGIS}

Events can be represented in a cube as circles (or other symbols) placed vertically according to the time of their occurrence. Figure 2 shows our implementation of the space-time cube display of events, which is available in the system CommonGIS [18]. In this implementation, the earliest events are at the bottom of the cube and the latest at the top. Variation of circle sizes or colors can additionally represent thematic characteristics of the events, for example, magnitudes of earthquakes. 


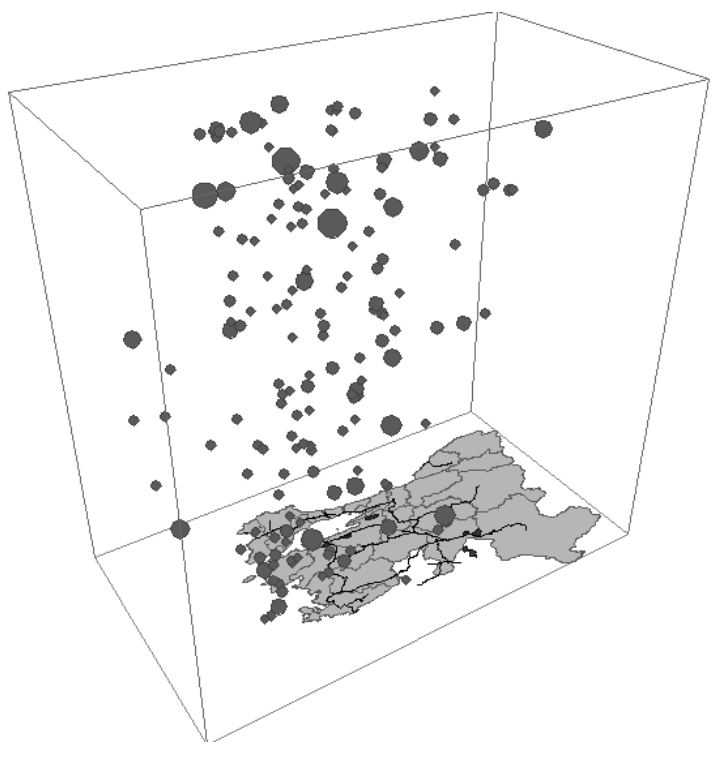

Figure 2. Data about events are represented in a "space-time cube". The vertical positions of the circles correspond to the times when the events occurred. Circle sizes or colors may reflect thematic characteristics of the events.

With this representation, a sequence of events that occurred around the same place shortly one after another would be translated into a chain of vertically aligned circles. In general, any three-dimensional shapes emerging due to visual proximity of event symbols deserve attention as an indication of possible event interactions, for example, inclined chains or conical structures. However, it should be borne in mind that visual grouping of symbols can be also a mere projection effect resulting from the representation of the threedimensional space on the two-dimensional computer screen. Therefore, our cube display allows the user to change her/his viewing perspective into the cube.

The time period under investigation may be rather long. "Pressing" the entire period into the height of the cube results in significant symbol overlapping and display cluttering, when hardly any pattern can be visible. Therefore, we made it possible to select a time subinterval and see only events that occurred during this time. The selected subinterval can be represented by the whole height of the cube available. The user can shift the subinterval along the time axis. In response, the scene is dynamically redrawn. As compared to temporal filtering on a map, the space-time cube display preserves the information about relative times of event occurrences. Another benefit is reduced overlapping of event symbols when several events occur in the same place at close but different times.

An important feature of the space-time cube presentation is its dynamic linking with a map as well as graphical displays of other types. This means that corresponding objects are identically marked in all displays when the user selects them with the mouse in one of the displays. This technique is illustrated in Figure 3: the user has selected a spatial cluster of events in the map and can see in the space-time cube how the events are distributed in time. In both the map and the cube these events are marked by circles with thick black borders. Similarly, it is possible to select event symbols in the cube display and see where these events are on the map or other displays.

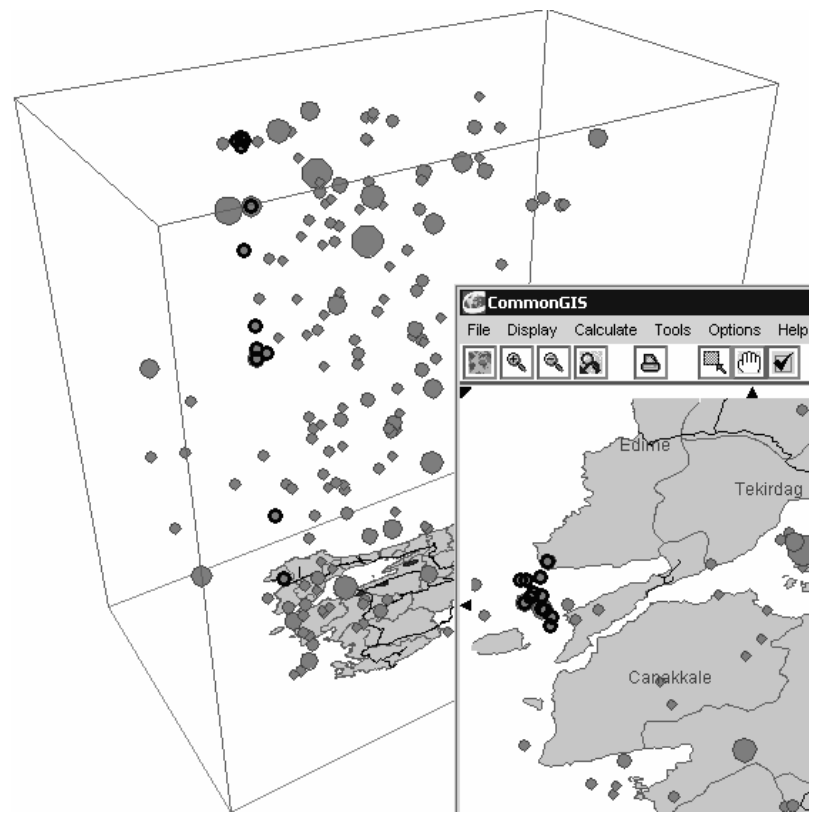

Figure 3. The "space-time cube" is dynamically linked to the map display by simultaneous highlighting of corresponding symbols.

For determining the time frame of some observed behavior pattern, such as vertical symbol alignment, it is convenient to use the movable plane that visually separates events having occurred before a specified time moment from later events. In Figures 2 and 3, this plane with the map drawn on it lies at the bottom of the cube but it can be lifted to the position corresponding to a user-selected moment. This technique also supports comparison of time frames of two or more events or patterns.

The space-time cube reacts to zooming and panning of the map and in this way supports zooming in the spatial dimension. Additionally, the space-time cube is linked to a dynamic query device that allows one to select events with specific characteristics, for example, only earthquakes with high magnitude. The diversity of mechanisms for linking various displays in CommonGIS is described in [19]. We stress tool linking as a central feature of the system, which allows users to carry out sophisticated data analyses. 
In the following section, we describe an example application of the tool to data about earthquake occurrences.

\section{Analysis of earthquakes in Marmara, Western Turkey}

The example dataset is a catalogue of earthquakes that occurred during the period from the beginning of 1976 until the end of 1999 in Marmara region (Western Turkey). The data have been previously cleaned, in particular, aftershocks removed. The catalogue contains 10550 events. Each data record includes the geographic location of an earthquake, the time of occurrence, and a few thematic attributes such as the depth of the epicenter, magnitude, intensity and some other seismic characteristics. An earthquake can be treated as an instant event: its duration is negligibly short in comparison to the whole time interval under investigation. Here, we are interested whether any spatiotemporal patterns can be observed in earthquake occurrence rather than in characteristics of individual earthquakes.

The major problem we encounter is that the earthquakes are too numerous. When we represent them all on a map, no spatial patterns can be seen but only a great number of overlapping earthquake symbols covering the whole territory. The map becomes suitable for data exploration only after applying temporal or other forms of filtering. The same problem appears when an attempt is made to display all 10550 earthquakes in a space-time cube. In this case, the height of the cube represents the whole 25 -year time period. The scale factor on the vertical axis has to be very small, and, as a result, many events have the same vertical position in the cube even when they are separated by quite a long time interval. Like the map, the cube is overfilled with overlapping event symbols. Hence, for seeing any patterns, temporal filtering becomes indispensable.

For temporal filtering, we use the temporal focusing control shown in Figure 4, top. We select a time interval of the length of about 4 months and let the system stretch this interval to the whole height of the cube. This significantly reduces symbol overlapping and makes the space-time cube display quite legible (Figure 4).

Now we can investigate the patterns formed by the earthquake symbols coming close together. When some bunch of circles attracts our attention, we check whether the closeness of the circles does not result from the projection. For this purpose, we select the circles by dragging a frame around them. The circles are switched to the "selected" state, i.e. marked by thick black borders, as is shown in Figure 3. After that, we change the viewing perspective and look whether the marked symbols still group together or dissipate. It should be admitted that the latter happens quite often. Thus, the symbol alignments that can be seen in Figure 4 do not actually correspond to event clusters: the alignments disappear after changing the viewpoint.

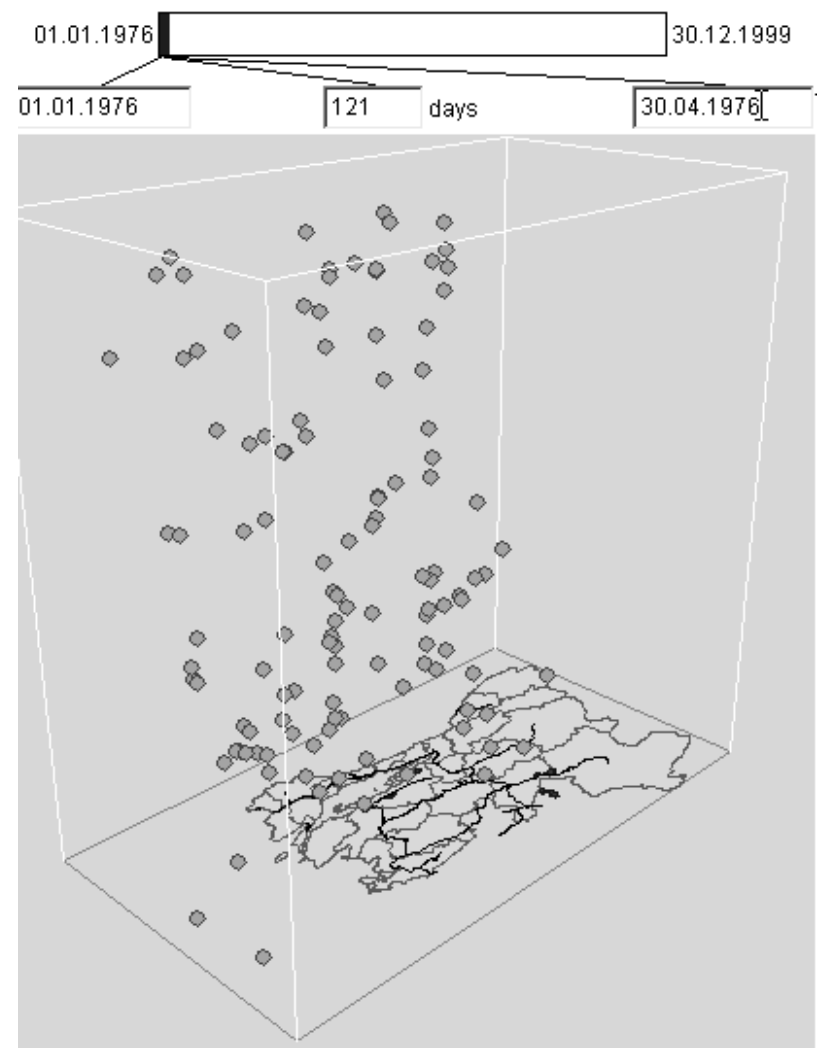

Figure 5. Temporal focusing applied to the spacetime cube display. The user has selected a 4-month time interval, which is now represented by the whole height of the cube.

However, we remember that the cube does not show us the whole dataset but only the initial 4 months of the 25-year period. We need to investigate the whole period. For this purpose, we slowly move the 4-month interval along the time bar (Figure 4, top). This results in the cube being dynamically redrawn. The visual effect is that the circles representing the earthquakes are slowly moving from top to bottom reminding a snowfall. If some interesting symbol arrangement emerges, we have sufficient time to notice it while it is "falling down". When some symbol bunch attracts our attention, we immediately stop moving the focus interval. Then we verify the finding by interactively selecting the symbols and altering the viewpoint. We also exploit the link between the cube and the map for refining our selection by means of deselecting spatially distant events.

In this manner, we eventually detect some potentially interesting clusters in space and time. Thus, Figure 5 shows a chain of events selected in the cube after its refinement with the help of the map. The same events are also marked on the map in Figure 6. 


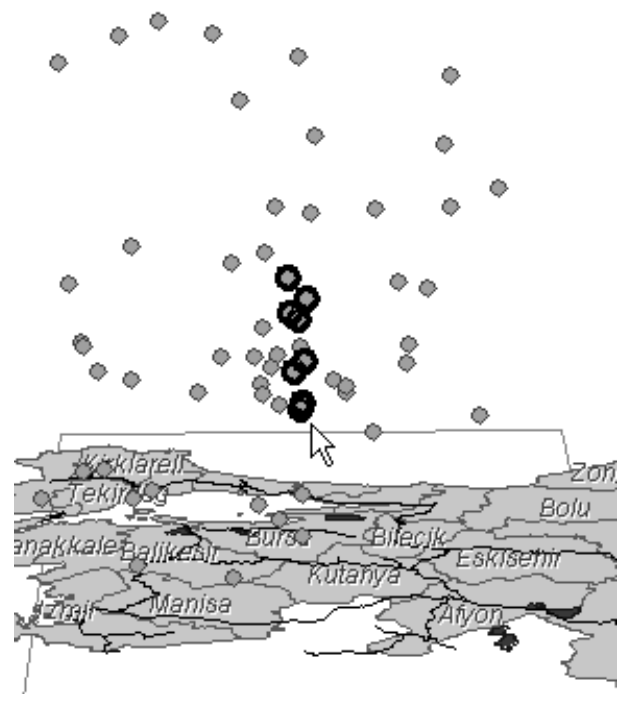

Figure 5. A probable spatio-temporal cluster has been detected and selected in the space-time cube. The circle in the middle of the chain has been unselected as irrelevant: the corresponding earthquake actually stands apart from the others and appears close only because of the projection.

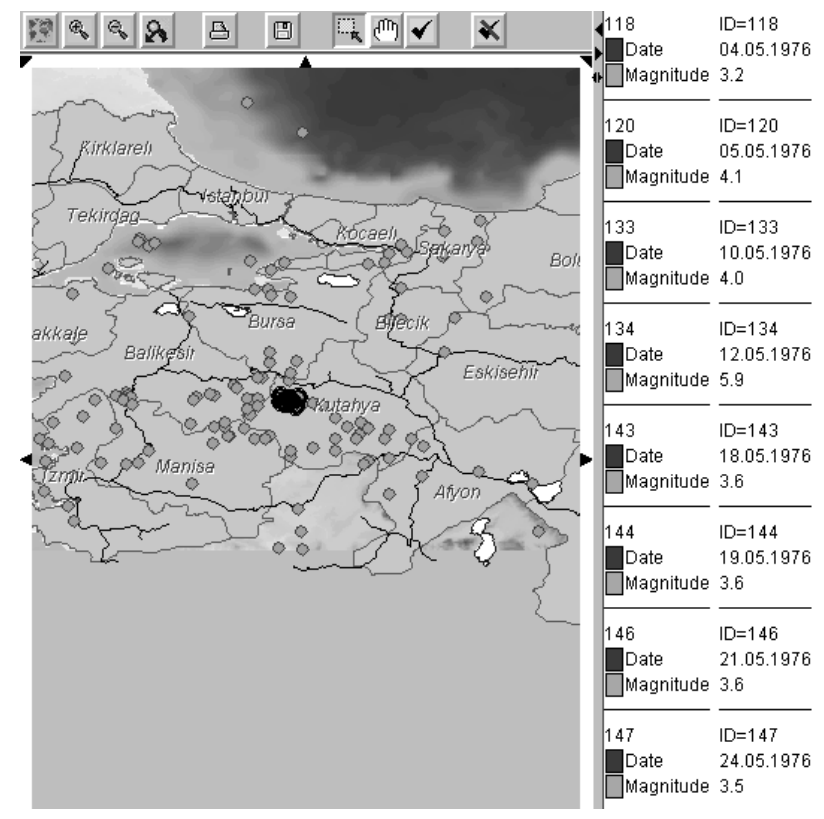

Figure 6. The same cluster of events as in Figure 5 is marked on a map. On the right, the dates and magnitudes of the selected events are displayed.

It can be clearly seen that the events form a spatial cluster on the territory of the province Kutahya (in the center of the map). On the right of the map, the dates and magnitudes of the selected earthquakes are shown. It may be noticed that all the earthquakes occurred during the time interval from May, 4, 1976 to May, 24, 1976. The strongest earthquake occurred on May, 12. Its magnitude was 5.9, and it was fourth in the sequence of events. It was followed by four weaker events.

The spatial and temporal closeness of the earthquakes suggests that there might be some interactions between these events or they might have a common origin. Unfortunately, we do not have sufficient domain knowledge for guessing about the meaning of this and other patterns. However, we believe that a seismologist would be interested to detect such sequences of earthquakes.

If we continue our exploration, we would find other potentially interesting groupings of earthquake occurrences in space and time, for example, a sequence of 7 earthquakes with magnitudes from 2.9 to 3.3 that occurred near Izmir between September 24 and October 6,1976 , or a chain of 6 earthquakes on the north of Kutahya province between the $1^{\text {st }}$ and the $20^{\text {th }}$ of July, 1990 . We can conclude that the tool potentially allows an analyst to find spatio-temporal event clusters. However, cluster detection in a real-size dataset occurs to be quite a laborious process requiring deep concentration.

\section{Conclusion}

Visual exploration of spatio-temporal data requires tools that can help users find answers to different types of questions that may arise in relation to such data. In this paper we described interactive space-time cube - a tool that represents time as an additional spatial dimension. We have found this analytical tool useful for visual exploration of spatio-temporal patterns of events. By an example of earthquakes, we have demonstrated that the space-time cube display allows one to detect sequences of events that occurred closely in space within short time intervals. However, the cube display would be less helpful without the link to a map by simultaneous highlighting of corresponding symbols. For exploration of long time intervals, temporal focusing is essential.

In our implementation, the cube display can also represent time-irrelevant numeric attributes of persistent spatial objects. In particular, we made some experiments with applying this visualization technique for multicriteria decision making problems. However, details of this study are out of the scope of this paper.

In our experiments with the tool we encountered some problems. Thus, during earthquake exploration, we have found it quite difficult to detect event clusters by applying only temporal focusing. The process required much time and deep concentration It was also quite difficult to select the right size of the "time window" for the focusing without having any expectations concerning the likely temporal extents of possible clusters.

To facilitate the work on pattern investigation, it would be good to provide some methods for automatic cluster detection under user's control. The user could specify, for example, certain thresholds for temporal and 
spatial "closeness" between events and a minimum cluster size, and the tool would try to find event subsets satisfying these constraints. The tool could also automatically search for periods of the overall high or low activity of the phenomenon.

We realize that usability studies should be carried out to check if the space-time-cube tool is understandable and convenient for users and effectively supports the analytical tasks it was designed for. We intend to do such studies in the future.

\section{Acknowledgements}

We are grateful to Prof. M.-J. Kraak (ITC, Netherlands) for interesting and fruitful discussions during the last years concerning visualization of spatiotemporal data. They gave us an inspiration for developing new and improving our existing analytical tools, in particular, the one described in the paper. We also thank Dr. Valeri Gitis and his colleagues from the Institute of Information Transmission Problems, RAS, Moscow, Russia, for the earthquake dataset used in our experiments. The presented work is partly supported by the European Commission projects GIMMI (IST-200134245; http://services.txt.it/gimmi/) and SPIN!-"'Spatial Mining for Data of Public Interest" (IST-1999-10536; http://www.ccg.leeds.ac.uk/spin/).

\section{References}

[1] T. Hägerstrand. What about people in regional science? Papers, Regional Science Association, 24, 7-21. 1970.

[2] N.R. Hedley, C.H. Drew, E.A. Arfin, and A. Lee. Hägerstrand Revisited: Interactive Space-Time Visualizations of Complex Spatial Data. Informatica: An International Journal of Computing and Informatics, 23 (2), 155-168. May 1999.

[3] What has ITC done with Minard's map? URL: http://www.itc.nl/personal/kraak/1812/minard-itc.htm

[4] M.-J. Kraak. The space-time-cube revisited from a geovisualization perspective. In Proceedings of the $21^{\text {st }}$ International Cartographic Conference (ICC) "Cartographic Renaissance", Durban, South Africa, 1988-1996. August 2003.

[5] N. Andrienko, G. Andrienko, and P. Gatalsky. Exploratory Spatio-Temporal Visualization: an Analytical Review, Journal of Visual Languages and Computing, special issue on Visual Data Mining, 14 (6), 503-541. 2003.

[6] D.J. Peuquet. It's about time: a conceptual framework for the representation of temporal dynamics in geographic information systems, Annals of the Association of American Geographers, 84 (3), 441-461. 1994.

[7] A.M. MacEachren. How Maps Work: Representation, Visualization, and Design. New York, The Guilford Press. 1995.

[8] T.A. Slocum. Thematic Cartography and Visualization, New Jersey, Prentice Hall, Uper Saddle River. 1999

[9] C. Blok. Monitoring Change: Characteristics of Dynamic Geo-spatial Phenomena for Visual Exploration, Ch. Freksa et al. (Eds.): Spatial Cognition II, LNAI 1849, Berlin Heidelberg, Springer-Verlag, 16-30. 2000.

[10] S.S. Stevens. On the theory of scales of measurement. Science, 103, 677-680. 1946.

[11] J. Bertin. Semiology of Graphics. Diagrams, Networks, Maps. (English translation by W.J. Berg). Madison, The University of Wisconsin Press. 1983. (original French edition: J. Bertin. Sémiologie Graphique. Paris, Mouton. 1967)

[12] A. Koussoulakou, M.J. Kraak. Spatio-temporal maps and cartographic communication. The Cartographic Journal, 29, 101-108. 1992.

[13] D. Stojanovic, S. Djordjevic-Kajan, A. Mitrovic, and Z. Stojanovic. Cartographic Visualization and Animation of the Dynamic Geographic Processes and Phenomena, in Proceedings of 19th International Cartographic Conference, vol. 1, 739-746. 1999.

[14] R. Edsall and D. Peuquet. A Graphical User Interface for the Integration of Time into GIS. Proceedings of the 1997 American Congress of Surveying and Mapping Annual Convention and Exhibition, Seattle, WA, 182-189. 1997.

[15] A. Fredrikson, C. North, C. Plaisant, and B. Shneiderman. Temporal, geographic and categorical aggregations viewed through coordinated displays: a case study with highway incident data. In Proceedings of Workshop on New Paradigms in Information Visualization and Manipulation (NPIVM'99), New York, ACM Press, 26-34. 1999.

[16] M. Harrower, A.L. Griffin, and A.M. MacEachren. Temporal Focusing and Temporal Brushing: Assessing their Impact in Geographic Visualization, In Proceedings of 19th International Cartographic Conference, vol. 1, 729-738. 1999.

[17] M. Harrower, A.M. MacEachren, and A.L. Griffin. Developing a Geographic Visualization Tool to Support Earth Science Learning, Cartography and Geographic Information Science, 27 (4), 279-293. 2000.

[18] G. Andrienko, N. Andrienko, and H. Voss. GIS for Everyone: the CommonGIS project and beyond, M.Peterson (ed.) Maps and the Internet, Elsevier Science, 131-146. 2003.

[19] N. Andrienko, G. Andrienko. Informed Spatial Decisions through Coordinated Views. Information Visualization, 2 (4), 270-285. 2003. 